\title{
A General Overview on Past, Present and Future Antimycotics
}

\author{
G. Van Minnebruggen ${ }^{*}, 1$, I.E.J.A. François ${ }^{2}$, B.P.A. Cammue ${ }^{2}$, K. Thevissen ${ }^{2}$, V. Vroome ${ }^{1}$, \\ M. Borgers ${ }^{1,3}$ and B. Shroot ${ }^{1}$ \\ ${ }^{I}$ Barrier Therapeutics n.v., a wholly owned subsidiary of Stiefel Laboratories, Geel, Belgium \\ ${ }^{2}$ Centre of Microbial and Plant Genetics (CMPG), Katholieke Universitiet Leuven, Leuven, Belgium \\ ${ }^{3}$ Department Molecular Cell Biology, Maastricht University, Maastricht, The Netherlands
}

\begin{abstract}
Since the discovery of amphotericin B in 1955 the armamentarium of antimycotic drugs now embraces many new chemical classes: azoles, allylamines and candins. However, despite the wide variety in chemical structure, there is a lack of diversity in terms of mechanism of action. The mechanism of action of the main classes of antimycotics as well as the therapeutic value of some representatives is discussed. Some challenges to innovation will be highlighted that when overcome will herald more effective therapeutic interventions. Finally, we will list antimycotics that are at a late stage of development.
\end{abstract}

Keywords: Candida spp., dermathophytes, antimycotics, fungicidal, drug development.

\section{INTRODUCTION}

During the past two decades the incidence of fungal infections has increased dramatically. This is especially the case in cancer patients, transplant recipients and patients with AIDS. In addition, patients receiving broad-spectrum antibiotics, corticosteroids, cytotoxic agents or parental nutrition are prone to fungal infection [1]. So far, notable advances in antifungal therapeutics were achieved with the development of less toxic formulations of amphotericin B, the introduction of improved triazoles, the advent of the echinocandin lipopeptides and the recent finding of broadspectrum benzoxaboroles.

In this review, we provide the reader with a general overview of the current classes of antimycotics and their principal applications, focusing on both systemic and topical fungal infections. For more in-depth literature regarding novel azole agents under development such as isavuconazole and the more recently introduced posaconazole, we refer to other excellent reviews in this regard [2-4]. In addition, we comment on some of the hurdles that need to be overcome in order to improve safety and pharmacokinetic profiles of today's antimycotics. Additionally, we will discuss the in vitro - in vivo enigma. Finally, some examples of tomorrow's antimycotics are listed.

\section{SURVEY OF THE MAIN CLASSES OF ANTIMYCO- TICS AND THEIR PRINCIPAL APPLICATIONS}

\section{Classes of Currently Used Antimycotics}

Systemic antimycotics are mostly reserved for the treatment of onychomycosis, tinea capitis, superficial and

*Address correspondence to this author at Barrier Therapeutics n.v., a wholly owned subsidiary of Stiefel Laboratories, cipalstraat 3, B-2440, Belgium; Tel: 32(0)497 3027 67;

E-mail: geertvanminnebruggen@myfastmail.com systemic candidiasis, and prophylaxis and treatment of invasive fungal infections [5]. Topical antimycotics are generally used for the treatment of superficial mycoses unless the infection is widespread or involves an extensive area or is resistant to initial therapy. The routinely used antimycotics for topical and systemic mycoses are grouped into eight different classes based on their mode of action [6,7]. Their respective mechanisms are depicted in Fig. (1). Azoles inhibit two cytochrome P450 (CYP) enzymes, lanosterol $14 \alpha$-demethylase and $22 \Delta$-desaturase, involved in the biosynthesis of the major lipid component of the fungal plasma membrane, ergosterol (Fig. 1A). The resulting ergosterol depletion and accumulation of precursor sterols alters the normal permeability and fluidity of the plasma membrane which impact the action of membrane-bound enzymes such as those involved in cell wall synthesis. Allylamines and thiocarbamates inhibit the early steps of ergosterol biosynthesis, more specifically squalene epoxidase, resulting in the accumulation of the sterol precursor squalene (Fig. 1A). High squalene levels may increase membrane permeability, leading to disruption of cellular organization. Polyene macrolides interact with ergosterol by forming a complex with its two hydrophobic side chains, resulting in the formation of pores leading to enhanced proton permeability, leakage of vital components and, ultimately, death of the organism (Fig. 1B). Fluorinated pyrimidines, such as 5-fluorocytosine (5-FC), interfere with DNA, RNA and protein synthesis in the fungal cells (Fig. 1C). Candins target the fungal cell wall enzyme complex $\beta$ 1,3-D-glucan synthase and as such inhibit the biosynthesis of the structural glucan component of the cell wall, which makes the cell vulnerable to osmotic lysis (Fig. 1D). Griseofulvin, only active against dermatophytes, acts via a different mechanism. It includes inhibition of the hyphal cell wall synthesis, binding to RNA, interference with nucleic acid synthesis and inhibition of microtubules essential for mitosis and cytoplasmic transport processes (Fig. 1E). Oxaboroles have the novel action of inhibiting fungal 
(F)

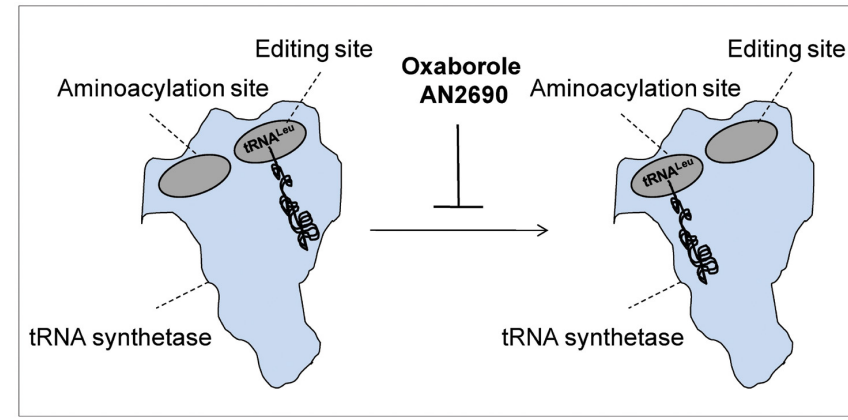

(E)

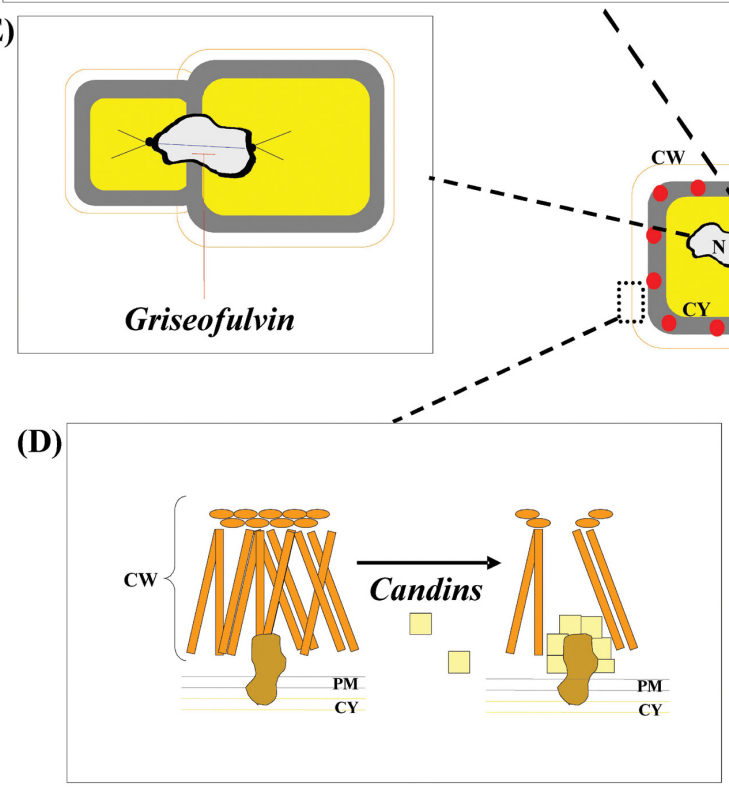

(A)

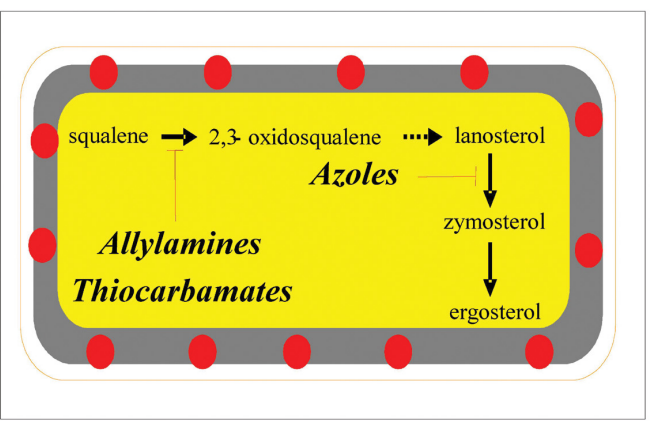

(B)

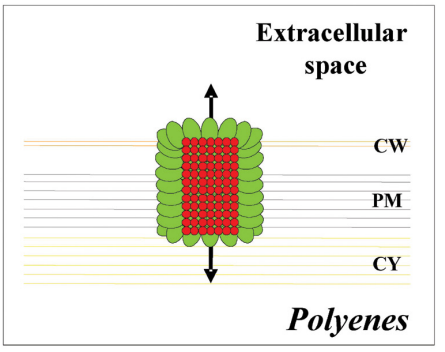

$$
\begin{array}{ll}
\text { : ergosterol } & \square \text { : candins } \\
\text { : polyenes } & \square \text { : cytosine permease } \\
\text { : tRNA } & \text { : tRNA synthetase }
\end{array}
$$

\section{$\|: \beta$-(1,3)-glucan $\quad \beta: \beta$-(1,3)-glucan synthase \\ o: $\beta$-(1,6)-glucan — : microtubules}

Fig. (1). Schematic representation of the mechanism of action of the different classes of antifungal agents. (A) Azoles, allylamines and thiocarbamates, (B) polyenes, $(\mathbf{C})$ 5-fluorocytosine, $(\mathbf{D})$ candins, $(\mathbf{E})$ griseofulvin and $(\mathbf{F})$ oxaboroles. $(\mathrm{CW}=$ cell wall, $\mathrm{M}=$ mitochondrion, $\mathrm{PM}=$ plasma membrane, $\mathrm{CY}=$ cytoplasm, 5-FU $=5$-fluorouracil, FUMP $=$ 5-fluorouridine monophosphate and FdUMP $=5$ fluorodeoxyuridine monophosphate).

cytoplasmic leucyl-tRNA synthetase by trapping tRNA ${ }^{\text {Leu }}$ in the editing site. This trapping of enzyme-bound tRNA ${ }^{\text {Leu }}$ prevents catalytic turnover, thus inhibiting synthesis of leucyl-tRNA ${ }^{\mathrm{Leu}}$ and consequently blocking fungal protein synthesis [4] (Fig. 1F).

In the following section, current treatment options for management of both types of mycoses (i.e. superficial and systemic) are discussed in more detail.

\section{Superficial Mycoses}

Superficial fungal infections are usually confined to the outer layers of skin, hair and nails. Generally, they are caused by dermatophytes (especially the genera Trichophyton, Microsporum and Epidermophyton), but also yeasts (e.g. Candida) and non-dermatophyte molds (e.g. Scopulariopsis brevicaulis) can be the infectious agents. In immune competent individuals, they usually have debilitating effects on a person's quality of life, whereas in the immune compromised population they can cause more complicated symptoms.

Physicians use topical antifungal agents as their firstchoice medication to treat dermatologic diseases caused by superficial infections. However, oral treatment is recommended in difficult to access infected areas such as toe-nails, large infected surface, immune compromised host or recurrent infection showing poor response to topical agents [2].

Topical formulations such as creams, aerosols, shampoos, lotions, gels and lacquers of azoles (e.g. miconazole, ketoconazole, econazole, tioconazole, oxiconazole, clotrimazole, bifonazole and sertaconazole), allylamines (e.g. terbinafine and naftifine), ciclopiroxolamine and amorolfine are available. They are indicated for treatment of superficial infections such as tinea corporis, tinea cruris, tinea pedis (also known as athlete's foot), pityriasis versicolor, 
onychomycosis (when nail involvement is $<50 \%$ and if no matrix involvement exists), cutaneous candidiasis, seborrheic dermatitis and Candida-associated diaper dermatitis [817]. For topical applications the choice of the vehicle to deliver the antifungal compound is of great importance and should be strongly linked to the condition of the involved and immediately surrounding non-involved skin areas. It is known for example that a more hydrophobic and protecting vehicle is better adapted to the diaper environment and that the right choice of formulation can shorten the treatment for example in seborrheic dermatitis.

Due to poor skin penetration profiles, topical antifungals are not ideal for (i) superficial infections of hair and nails, such as tinea capitis, tinea barbae, tinea unguium and onychomycosis (when nail involvement is $>50 \%$ ), and (ii) in skin infections in which these topical antimycotics give no to little cure after one or two weeks therapy [2,13,18-20]. In these cases, oral rather than topical, administrated azoles and allylamines are recommended [13,15]. Additionally, oral griseofulvin can be used as an alternative therapy if the infection is caused by dermatophytes [21].

The importance of onychomycosis is often underestimated as it affects the quality of life of the patient. Far beyond being a simple "cosmetic problem", infected nails can serve as chronic reservoirs for infection or colonization which can give rise to recurrent skin mycoses [22]. Since onychomycosis represents such an important superficial mycosis, we will go into more detail in its treatment options. For about 40 years, griseofulvin was the only antifungal agent available for treatment of onychomycosis. However, its rather limited efficacy, its poor pharmacokinetic profile and its high recurrence rate prompted researchers to seek more effective drugs. Currently, three agents, terbinafine, itraconazole and fluconazole, are used as oral therapy for the treatment of onychomycosis $[15,23]$. Although oral monotherapy is effective in onychomycosis, oral regimens in combination with topical adjuvant therapy is considered to improve the therapeutic outcome [24,25]. Currently used combination therapies comprise combinations of oral terbinafine or itraconazole with 5\% amorolfine nail lacquers [26]. Another type of film forming presentation containing ciclopirox $\left(\mathrm{Penlac}^{\mathrm{TM}}\right)$ is used as topical treatment in immune competent patients with mild to moderate onychomycosis of fingernails and toenails without involvement of lunula due to Trychophyton rubrum [27]. AN2690 (5-fluoro-1,3-dihydro1-hydroxy-2,1-benzoxaborole), a novel boron-containing small molecule designed to penetrate nails, is believed to be a potential successor of ciclopirox lacquers. As discussed later, this drug, with much better nail penetration kinetics than ciclopirox, has been shown to have broad spectrum antifungal activity in vitro and is currently in clinical trials to treat onychomycosis topically [28-30].

\section{Systemic Mycoses}

During the past 20 years, the incidence of systemic mycoses in humans has increased dramatically, especially due to the growth of the immune compromised patient population and the increased use of invasive devices (such as central venous catheters) and implants (such as prosthetic cardiac valves). A number of surveys have revealed that in the beginning of 2000 more than $50 \%$ of Candida infections were caused by $C$. albicans [31]. However, non-Candida albicans spp. such as C. glabrata are being increasingly isolated as causal agent for systemic mycoses [32]. Apart from Candida spp., infections by yeasts such as Cryptococcus neoformans as well as by filamentous fungi (moulds) such as Aspergillus spp., Fusarium spp., Scedosporium spp., Penicillium spp. and Zygomycetes spp. are also increasingly reported since 2000 [33-35]. Besides, a myriad of pathogens, such as e.g. Pneumocystis jiroveci, Histoplasma capsulatum, Paracoccidioides brasiliensis and Coccidioides immitis, are in rise [36-39]. Strikingly, mortality rate associated with systemic Candida and Aspergillus infections still sticks high, $35-64 \%$ for Candida up to $100 \%$ for Aspergillus in organ transplant recipients [40-42].

The basis of systemic mycoses management goes back to the 1940's with the discovery of the antifungal activity of sulfonamides. During the two decades that followed, amphotericin B (which is still considered to be the gold standard for the treatment of most life-threatening mycoses) became available. Since then, the following drugs were approved: 5fluorocytosine (early 1960s), intravenous miconazole and oral ketoconazole (late 1970s), fluconazole and itraconazole (1980s), caspofungin (2001), voriconazole (2002) and micafungin (2005). Today these drugs, grouped by chemical class as, fluorinated pyrimidines, polyenes, azoles and candins, are still used in practice for several types of systemic mycoses. Some specific examples are listed below:

\section{(i) Fluorinated Pyrimidines}

5-fluorocytosine $\left(\right.$ Ancotil $^{\mathrm{TM}}$ ) is used to treat systemic mycoses caused by Candida spp., Cryptococcus spp. and Aspergillus spp. [43]. Intrinsic resistance in Candida spp. to this molecule is uncommon [44], though as with other antimetabolites the emergence of drug resistance can be a problem. Therefore, 5-fluorocytosine is preferentially used in combination with another antifungal agent. Along with Amphotericin B, 5-fluorocytosine is known as a premium combination therapy for the treatment of Cryptococcus meningitis [45].

\section{(ii) Polyenes}

Amphotericin B deoxycholate (AmB; Fungizone ${ }^{\mathrm{TM}}$ ) is a polyene with a very broad spectrum of activity including most yeasts and filamentous fungi [34]. It is useful in treating candidiasis, cryptococcosis, histoplasmosis, blastomycosis, aspergillosis as well as zygomycosis $[46,47]$. Side effects are common, occurring in $50-90 \%$ of cases and are principally nephrotoxicity or infusion-related. However, there is rationale that the renal toxicity usually associated with longterm use of $\mathrm{AmB}$ deoxycholate is reduced or even absent when liposomal formulations of $\mathrm{AmB}$ are used as treatment regimen: liposomal $\mathrm{AmB}$ (L-AmB), $\mathrm{AmB}$ lipid complex (ABLC) and AmB colloidal dispersion (ABCD). L$\mathrm{AmB}$, rather then $\mathrm{AmB}$, has become the standard therapy [46].

\section{(iii) Azoles}

Fluconazole (Diflucan ${ }^{\mathrm{TM}}$ ) is available in oral or intravenous formulations and is well tolerated, with fewer drug interactions than other azoles. It is inactive against nondermatophyte filamentous fungi. Itraconazole (Sporanox ${ }^{\mathrm{TM}}$ ) has a broader activity spectrum than fluconazole. It is active 
against yeasts and moulds with the exception of Fusarium spp., Scedosporium spp. and the Zygomycetes. Although some precaution with prescription of this drug is required in patients with evidence of ventricular dysfunction such as congestive heart failure, both oral and intravenous formulations are widely used for non-immuno compromised patients [48]. The spectrum of voriconazole $\left(\mathrm{Vfend}^{\mathrm{TM}}\right.$ ) is similar to that of itraconazole, but includes several emerging moulds such as Fusarium spp. and Scedosporium spp. [49]. Voriconazole may require some precaution since it is prone to metabolization by the cytochrome (CYP) P450 enzyme system and therefore has potential drug interactions [50]. In Europe, both oral and intravenous formulations of voriconazole are licensed for the treatment of fluconazole-resistant invasive Candida infections such as C. krusei. Furthermore, voriconazole is presently the drug of choice against invasive aspergillosis [51]. For an overview on the activity spectra of the comparator azole drugs fluconazole, itraconazole and voriconazole we refer to a study of Sabatelli and colleagues that included 19000 clinically important strains of yeasts and molds collected from 200 medical centers worldwide over a 10-year time span [52].

\section{(iv) Candins}

Caspofungin (Cancidas ${ }^{\mathrm{TM}}$ ) is used as an intravenous formulation for the treatment of invasive Candida spp. and Aspergillus spp. infections [53]. It has good activity against C. glabrata but $C$. parapsilosis may respond less to treatment [54]. Lack of susceptibility is also reported for important basidomycetes such as Cryptococcus spp., Rhodotolura spp. and Trichosporon spp. [2].

\section{Fungicidal versus Fungistatic Action}

It is essential to treat patients with life-threatening mycoses with broad-spectrum fungicidal antimycotics, as early initiation of effective systemic antifungal treatment is essential for a successful clinical outcome in these patients. However, clinical clues for diagnosis are sparse and early microbiological proof of e.g. invasive aspergillosis is rare. For such patients, it is essential to treat them with broadspectrum fungicidal antimycotics. In practice, the new generation of azoles like voriconazole and posaconazole, as well as amphotericin B or caspofungin are used to treat such patients [55]. However, while amphotericin B is fungicidal against filamentous fungi, including Aspergillus spp., it is also characterized by a high cytotoxicity in general [56]. Echinocandins like caspofungin are rather fungistatic against Aspergillus spp. and their complex structure results in high production costs [57]. In contrast to the fungistatic activity of triazoles against Candida spp. (including itraconazole and voriconazole), these azoles are fungicidal against Aspergillus spp. [58].

\section{CURRENTLY USED ANTIMYCOTICS WITH MULTI- PLE MECHANISMS OF ACTION}

\section{Antimycotics with Additional Mode of Antifungal Action}

There exists some heterogeneity in the mode of action amongst azole antifungals. Besides ergosterol biosynthesis inhibition, the earlier azoles (such as miconazole and ketoconazole) are reported to have a more complex mode of action, inhibiting several membrane-bound enzymes as well as membrane lipid biosynthesis [59]. Interestingly, miconazole exerted a prominent effect on enzymes involved in the production and breakdown of reactive oxygen species (ROS) [60-62]. The production of ROS together with the simultaneous inhibition of peroxidative defence enzymes has been held responsible for miconazole's fungicidal action against $C$. albicans [44]. Moreover, miconazole was recently demonstrated to induce stabilization of the actin cytoskeleton in yeast prior to induction of intracellular ROS accumulation [63]. Additionally, the complexity of the mode of action of azoles is further demonstrated by the induction of farnesol production in susceptible Candida spp. upon incubation with fluconazole, ketoconazole, clotrimazole and miconazole [64]. Not only azoles, but also polyenes were demonstrated to have an additional mode of antifungal action. More specifically, amphotericin B was shown to induce increased endogenous ROS levels in C. albicans and concommitantly programmed cell death or apoptosis [65].

\section{Ancillary Properties of Azoles}

Cutaneous fungal infections are often superinfected by bacteria. Therefore, antifungals should preferentially possess antibacterial activities, especially when destined for topical application. This is the case for the azoles miconazole and ketoconazole which exert antibacterial activities. Miconazole is bactericidal against gram positive bacteria such as Propionibacterium acnes and Staphylococcus aureus at low concentrations, while ketoconazole has only a bacteriostatic activity at high concentrations [66].

Fungal skin lesions are mostly accompanied by inflammatory reactions, hence concomitant anti-inflammatory activity as an adjunct property in one molecule may be important. Although there are indications that ketoconazole has some inflammatory activity, glucocorticosteroids are often incorporated in antifungal ointments to achieve antiinflammatory effect.

Fungal infections are often favored by excessive sebum production. Indeed, growth of Malassezia spp. and maintenance of seborrhoeic dermatitis and pityriasis versicolor are highly dependent on lipid production [67]. Consequently, drugs that, ancillary inhibit the production and flow of sebum are highly desirable for the treatment of these cutaneous diseases.

\section{Adjuvant Therapy}

It is known that the simple modification of the calcium concentration in culture media highly influences the antifungal activity of azoles. In fact, calcium addition decreases their activity whereas chelating this cation increases its activity [68]. This observation led to the combined use of azoles with calcineurin inhibitors such as cyclosporine A and tacrolimus. The effect was synergistic and resulted in potent fungicidal activity against C. albicans [69].

\section{HURDLES TO OVERCOME IN THE SEARCH FOR NEW ANTIMYCOTICS}

Although today's approved antimycotics such as the widely prescribed azoles possess a broad activity spectrum, 


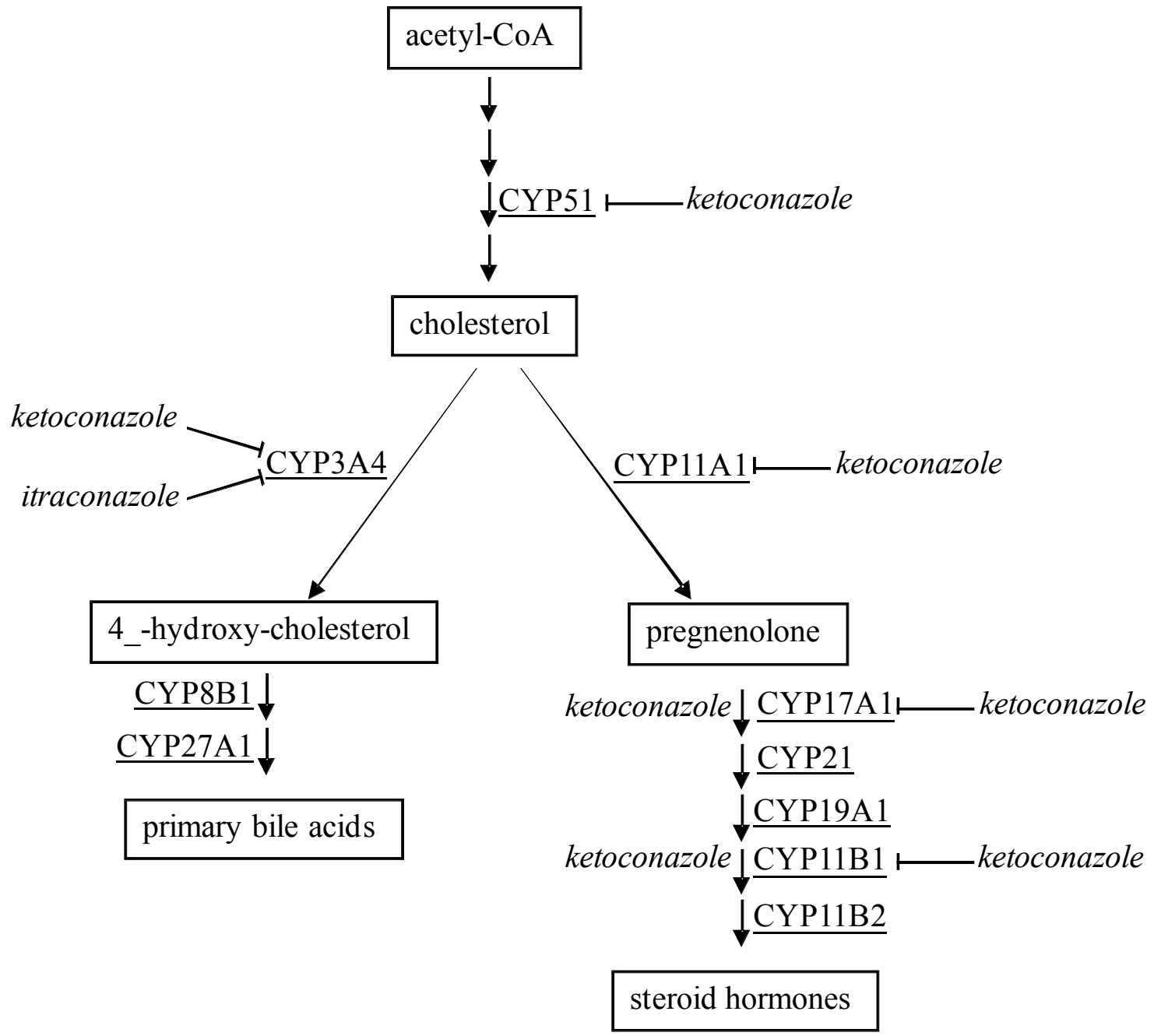

Fig. (2). Schematic overview of cholesterol metabolism, focusing on the pathways that can be inhibited by ketoconazole and itraconazole. In boxes key molecules of cholesterol metabolism are given. The different CYP enzymes responsible for the different enzymatic steps are shown underlined. Inhibition of CYP enzymes by azoles is indicated.

relatively favorable kinetics and low toxicity, their properties are subject to improvement. To achieve in the near future key improvements three main areas of research are actively pursued:

i. Enhanced fungicidal action

ii. Reduced incidence of resistance

iii. Greater selectivity for inhibiting fungal CYP isoforms.

\section{Safety Profile}

Current azole antifungals (e.g ketoconazole, itraconazole, miconazole and fluconazole) can inhibit both fungal and mammalian CYPs. At concentrations $>100 \mathrm{nM}$, ketoconazole inhibits the fungal CYP51, but also the mammalian CYP51 that plays an important role in cholesterol biosynthesis (Fig. 2). Additionally, at these higher concentrations, ketoconazole also affects the activity of enzymes involved in catabolism of cholesterol. More specifically, ketoconazole inhibits 17-hydroxylase-17,20-lyase (CYP17), the cholesterol side chain cleavage enzyme (CYP11A1), and the 11ßhydroxylase (CYP11B1) (Fig. 2) [70]. Itraconazole is almost devoid of effects on steroid metabolism [71]. However like ketoconazole, itraconazole inhibits CYP3A4, a major drugmetabolizing $\mathrm{P} 450$ isoform that also plays a role in cholesterol metabolism in the human liver [72]. The $50 \%$ inhibitory concentrations (IC50s) of ketoconazole and itraconazole for CYP3A4 inhibition were 11.7 and $32.6 \mathrm{nM}$, respectively [73]. Fluconazole and miconazole are potent inhibitors of CYP2C9, which plays a major role in the metabolism of drugs such as phenytoin, S-warfarin and a range of nonsteroidal anti-inflammatory drugs [74,75]. The search for future azole antifungals that show pronounced selectivity for fungal CYP's will be advantaged by the recent research related to the production of strains of $S$. cerevisiae, humanized with respect to the amino acids encoded at the CYP 51 (ERG11) yeast locus [76].

AmB (still the mainstay of therapy for some serious infections), is besides its extreme potency also quite famous for its renal toxicity and infusion-related side-effects [77]. Dose-limiting nephrotoxicity directly relates to the mode of action of $\mathrm{AmB}$ when it selectively binds to fungal ergosterol but also, to a lesser extent, to human membrane-associated low density lipoproteins such as cholesterol. On the other hand, toll-like receptor 1 and 2 play a major role in the infusion-related, immunomodulatory side-effects [76]. 


\section{Resistance Development}

To date, several mechanisms have been proposed to explain azole resistance of $C$. albicans, including (i) reduced accumulation of the azole due to reduced uptake or due to an active transport out of the cell, (ii) overproduction or mutation of the target enzyme [78] and (iii) the increased occurrence of biofilms.

Interactions between sterols and phospholipids in the cytoplasmic membrane affect membrane fluidity and asymmetry and consequently influence the transport of substances across the membranes. A decrease in azole uptake by the fungal cell may result from changes in the sterol and/or the phospholipid composition of the fungal cell membrane. Hence, intracellular accumulation of azoles can be reduced by the lack of drug penetration because of low ergosterol levels. However, an important cause of reduced intracellular accumulation of the drug is the active transport of the drug out of the cell. In $C$. albicans, two families of drug efflux pumps are described: ATP-binding cassette transporters and major facilitator proteins.

(ii) Upregulation of the ERG11 gene, which encodes the major target enzyme of the azoles lanosterol 14- $\alpha$ demethylase, has been observed in azole-resistant $C$. albicans and C. glabrata isolates [79-81]. However, other studies have reported no significant change in expression levels of the ERG11 gene in azoleresistant clinical isolates of $C$. glabrata $[82,83]$. This indicates that overexpression of the ERG11 gene probably is not critical for the development of azole resistance. Direct evidence for certain mutations of Erg $11 p$ resulting in decreased affinity to the drug was provided by biochemical analysis of heterologously expressed enzymes. The affinity of fluconazole for lanosterol 14- $\alpha$-demethylase containing the mutations $\mathrm{Y} 132 \mathrm{H}, \mathrm{G} 464 \mathrm{~S}$ or $\mathrm{R} 467 \mathrm{~K}$ was reduced as compared with the wild-type enzyme, confirming that these naturally occurring mutations indeed caused drug resistance in clinical C. albicans isolates $[56,84,85]$.

(iii) Another emerging source of antifungal resistance is the occurrence of a biofilm. Biofilms are extracellular matrices produced by microbes themselves. They serve to help organisms attach to living or non-viable surfaces. Presence of biofilms may markedly reduce the susceptibility of a microbe to antimicrobial agents by either reducing the accessibility or more fundamentally to the phenotypic change undergone by the microbe $[86,87]$. Recent surveys estimate that to date about $65 \%$ of all human microbial infections involve biofilms and that since 2005 the majority of invasive diseases produced by $C$. albicans are associated with biofilm growth [88-96]. The most important phenotype is a reduced susceptibility to conventional antimycotics and to the host immune system [97-102]. In 2004, Mateus and co-workers demonstrated that drug efflux pumps play a role in the drug resistance of early biofilms [82,103]. In contrast, resistance of mature biofilms does not rely on the known antifungal efflux pumps [82,84]. It has been hypothesized that a change in membrane sterol composition during biofilm formation might explain resistance to amphotericin $\mathrm{B}$ and the azoles. In addition, the MAPK Mkclp seems to be a regulator of azole resistance in mature biofilms [104].

Like Candida spp., Aspergillus spp. can be prone to azole resistance. Multiple mechanisms are known to be responsible, with differing degrees of azole-cross resistance, including mutations in the CYP51A gene at positions 54, 98, 138, 220 and 448 [105]. Although efflux pump-mediated resistance also occurs in Aspergillus spp. as in Candida spp., most often CYP mutations underly azole-resistance in Aspergillus spp. [2].

Besides azole resistance, it is worth to mention that candin resistance is frequently reported in clinical isolates of C. albicans, C. glabrata, C. krusei and C. tropicalis. Here, the resistance phenotype is associated with amino acid substitions in two 'hot-spot' regions of Fks1, the major subunit of glucan synthase [106]. The Fks1 mutations are known to be genetically dominant and confer crossresistance to all echinocandin drugs. First evidence exists that Fsk1 mutations can cause resistance in yeast as well as in moulds such as Aspergillus fumigates, and that this mechanism may be pervasive in fungal kingdom [105].

\section{Pharmacokinetics}

Optimal active drug concentration at the site of infection is another important target for improved therapeutics. Subtle structural modifications may result in striking differences in plasma pharmacokinetics, as in case of $\mathrm{AmB}$ and its lipid formulations $\mathrm{L}-\mathrm{AmB}, \mathrm{ABLC}$ and $\mathrm{ABCD}$. The latter three derivatives display pharmacokinetic profiles that are influenced by diverse disposition of respective lipid moieties, whereas liberated AmB its pharmacokinetic behavior is independent of lipids [107]. Triazoles such as e.g. fluconazole, itraconazole, voriconazole, pramiconazole, posaconazole, albaconazole, ravuconazole and isavuconazole are to a certain extent so-called 'look-alikes', though they differ from each other in species-specificity, potency as well as in their pharmacokinetics. Compared to the oldies fluconazole and intraconazole, the newer triazoles have in general an improved specify for fungal CYP, a different metabolic profile and a longer half-life in blood [106-109]. Metabolisation in the liver where the cytochrome P450 (CYP) system is involved is frequent for all triazoles and requires some precaution during patient management. This alertness is not required for candins although they are also rapidly taken up by pheripheral tissues such as the liver. In the first 24h, this liver uptake appears to be the main route of elimination from plasma for all three candin prototypes, i.e. caspofungin, micafungin and anidulafungin [106].

\section{THE IN VITRO - IN VIVO ENIGMA}

The minimum inhibitory concentration (MIC) of an antifungal drug as determined in vitro is expected to predict and correlate with the behavior of that drug in infected tissues. Indeed, manufacturers of antimycotics often quote low MIC values in their communication as implied evidence of therapeutic potency. In reality, however, the correlation is not always so straightforward. When multiple reports of the 
correlation of therapeutic outcome with in vitro susceptibility are examined (reviewed in [110]), a pattern commonly referred to as the '90-60 rule', emerges. The 9060 rule observes that infections due to susceptible isolates respond to appropriate therapy approximately $90 \%$ of the time, whereas infections due to resistant isolates (or infections treated with inappropriate antibiotics) respond approximately $60 \%$ of the time. Why does this response follow the 90-60 rule rather than a 100-0 rule? Some possibilities exist to explain this. First, the in vitro susceptibility might vary if a different testing method is used. For example the standard Clinical and Laboratory Standards Institute (CLSI) broth dilution techniques for antifungal susceptibility testing use planktonic populations, and, therefore, do not enable true evaluation of antifungal efficacy against Candida biofilms. Additionally, the CLSI method may not be the method of choice for the evaluation of slow growing organisms. In the latter case, the method of the European Committee on Antimicrobial Susceptibility Testing (EUCAST), is highly recommended. Both, CLSI and EUCAST (two microdilution methods) have made it possible to identify break points (so called MIC values) for specific antifungals against specific test-organisms [111,112]. Amongst others, determination of fungicidal activity, flow cytometry, ergosterol quantification, colorimetric microdilution, disk diffusion and agar dilution methods are also in use for susceptibility testing. No surprise that also between those methods subtle differences may exist. A popular commercial method in use today to overcome the problem of the different conditions between different labs when measuring MIC values are the so-called E-test strips [111]. In principle, those strips are based on a combination of the concepts of the dilution and diffusion tests. Like dilution methods, E-test directly quantifies antifungal susceptibility in terms of discrete MIC values. As the E-test consists of a predefined and continuous concentration gradient, the MIC values obtained can be more precise than values from conventional procedures based on discontinuous two-fold serial dilutions [113].

Second, the problem might be linked to inter-patient variability in terms of pharmacokinetics, lack of host response or production of toxins. Hence, individual susceptibility testing should be considered as a part of the process of predicting whether a given patient will respond to therapy. In essence research into the development of diagnostics is advocated.

Third, some caution should be exercised in the case of the assessment of the in vitro efficacy of imidazole antifungal agents using MIC determination. In practice these data are subject to large inter-laboratory variability. Notable variables include the nature of culture medium, temperature, $\mathrm{pH}$, duration of incubation, inoculum size, phase of fungal growth, the presence of serum, leukocytes, cornified cells and keratins [114-116]. Keratins for example were found to affect the terbinafine efficacy in a clinically relevant onychomycosis in vitro test model. Indeed, the terbinafine concentration needed to block invasive mycelia that were formed after inoculation of human nail powder with Trichophyton rubrum was found to be $1 \mu \mathrm{g} / \mathrm{ml}$ after 4 weeks exposure, which is much higher than MICs $\leq 0.03 \mu \mathrm{g} / \mathrm{ml}$ in standard NCCLS MIC assays techniques [92]. Accordingly, in vitro results should be interpreted with caution and some in vitro susceptibility test results may poorly reflect in vivo efficacy of the imidazole drugs [117]. Ketoconazole, the first imidazole used for oral treatment, represents a notable example. Its observed in vivo efficacy in experimental models of dermatomycoses and candidiasis is distinctly superior than might be expected from its in vitro activity against the same species [93].

\section{EMERGING ANTIMYCOTICS}

Using the following selected examples we illustrate that the hunt for novel, improved antimycotics with better efficacy and safety profiles and less drug-drug interactions is very active.

In addition to caspofungin (Cancidas ${ }^{\mathrm{TM}}$, Merck\&Co), two new echinocandins, micafungin (Mycamin $\mathrm{e}^{\mathrm{TM}}$, Fujisawa Healthcare) and anidulafungin (Eraxis ${ }^{\mathrm{TM}}$, Pfizer), appeared in 2005 and 2006, respectively [118-121]. Primarily, these echinocandins are designed for the treatment of deep mycoses. At present, it is not yet clear whether they will be used to treat dermatophytes infections.

Voriconazole $\left(\right.$ Vfend $^{\mathrm{TM}}$, Pfizer) and posaconazole (Noxafil $^{\mathrm{TM}}$, Schering-Plough) are two broad spectrum triazole antifungal agents that were recently approved. In common with other azoles, they act principally by inhibition of fungal CYP51 [122,123]. Currently, voriconazole is approved for a multitude of indications (i.e. invasive aspergillosis, candidemia in non-neutropenic patients, disseminated candidal infections, esophageal candidiasis and infections caused by Scedosporium apiaspermum and Fusarium species), whereas, to our knowledge, posaconazole is only approved for two indications (i.e. prophylaxis of invasive Aspergillus and Candida infections in high-risk patients and first in-line treatment for candidiasis). Clinical studies in dermatophyte infections are ongoing.

Ravuconazole (Bristol-Myers Squibb) is another new member of the azole family that acts selectively on the fungal CYP51 [124]. Since this triazole has not yet advanced to phase 3 clinical trials, it is too early to predict whether or not this drug will reach the market and, if so, for which specific indications. Considering its antifungal spectrum, ravuconazole would be a good candidate for treatment of non-Candida albicans infections, including dermatophyte infections [125-128].

Hyphanox $^{\mathrm{TM}}$, a late phase drug candidate of Stiefel Laboratories, is an oral formulation of itraconazole (a melt extrusion form) being developed for the treatment of various fungal infections, including vaginal candidiasis, tinea pedis and onychomycosis. It is anticipated that this $200 \mathrm{mg}$ tablet formulation will improve patient compliance and reduce pharmacokinetic variability that is frequently encountered with current therapies. Apart from fluconazole, most azoles can show high inter-patient variability in terms of serum AUC and $\mathrm{C}_{\max }$ levels. A notable food effect is also observed.

Pramiconazole (Stiefel Laboratories) is a new member of triazole class which is in phase 2 development for the treatment of superficial infections caused by dermatophytes (e.g. Trichophyton spp., Microsporum canis and Epidermaphyton), yeasts (Candida spp. and Malassezia spp.) and many other fungi $[18,88]$. At present, seven Phase $2 \mathrm{a}$ and 
one Phase $2 \mathrm{~b}$ studies are completed. These studies comprise a pilot and a dose-finding trial in pityriasis versicolor and pilot trials in seborrheic dermatitis, tinea pedis, tinea cruris and tinea corporis [12,18,129-132]. In all studies, both primary and secondary endpoints were met. A drug efficacy study for use in onychomycosis is in progress.

Albaconazole (Laboratorios Uriach \& Cía. S.A.) is a new triazole with a potent, broad spectrum of antifungal activity (inclusive Candida spp., Cryptococcus spp. and Aspergillus spp.), good pharmacokinetics, low toxicity and excellent oral bioavailability (nearby $80 \%$ in rats and $100 \%$ in dogs) [133].

Posaconazole is a member of the azole class of antifungals recently approved for the prophylaxis and treatment of invasive fungal infection. It has proven fungistatic activity against most Candida spp., Cryptococcus spp. and Trichosporon spp, and, on top, has superior activity to the other azoles against Zygomycetes isolates [134].

Basilea Pharmaceutica Ltd selected a new broad spectrum azole prodrug (BAL8857) that has the potential to treat mucocutaneous and invasive fungal infections as well as onychomycosis $[135,136]$. After oral or intravenous administration, BAL-8557 is rapidly cleaved into isavuconazole, in a reaction catalysed by human plasma esterases. Being a broad spectrum water-soluble drug, BAL8857 was in 2008 the subject of two phase III studies that investigated the safety, tolerability and efficacy in the prevention of fungal diseases and the pharmacokinetics of the antifungal drug, namely NCT00413439 and NCT00444366 [134].

Abafungin (Abasol ${ }^{\mathrm{TM}}$, York Pharma) is a promising drug candidate of the arylguanidine family that possess fungicidal activity against dermatophytes, yeasts and moulds, regardless of whether the etiologic agents are in the growth or dormant phase [137]. A $1 \%$ cream formulation has reached an advanced stage of development and may be indicated not only for Candida intertrigo and tinea infections, but also for skin diseases caused by yeasts such as pytiriasis versicolor.

The new imidazole derivative luliconazole (Nihon Nohyaku Co. Ltd.) showed promising in vitro and in vivo activity when compared to bifonazole, terbinafine and lanoconazole. Besides many yeast species, luliconazole is active against several dermatophyte- and mold species $[138,139]$. In line with luliconazole, some 2,4,5-tri-substituted imidazole derivatives from Lorus Therapeutics have antifungal activity against $C$. albicans.

Anti-invasins (Microbia) represent a class of broad spectrum antifungals that have been selected using a morphogenetic transformation assay in C. albicans [140].

Sordarins (Diversa Corporation) were recently selected as naturally derived antifungal compounds with a novel mode of action. Interestingly, they show activity against azole-resistant fungi [116].

AN 2690 is the novel benzoxaborole (Anacor) in clinical development that shows promising preliminary results. In a reported trial, 60 subjects with mild to moderate, $\mathrm{KOH}$ positive, distal subungual onychomycosis were treated with either AN2690 5.0\% or $7.5 \%$ once daily for a 6 month treatment period followed by a 6 month treatment-free follow-up period [27].
Efungumab (formerly know as Mycograb) is a monoclonal antibody targeting heat shock protein 90 (HSP90). In vitro and first clinical data show that Efungumab shows activity against Candida spp. when used alone and synergism when combined with fluconazole, caspofungin, and amphotericin B. Although further safety data are needed, it looks like Efungumab may become a new antifungal agent with unique mechanism of action for treatment of invasive candidiasis [141].

\section{CONCLUSIONS}

Over the last fifty years, several important discoveries have lead to the current approved armamentarium of antimycotics, including polyenes, imidazoles, triazoles, allylamines and candins. Our review of representative examples of these classes of drugs, illustrates their therapeutic value in the fight against many pathogenic fungi. Failure rates in management of fungal infections are still significant, consequently it is not surprising that there is still an unmet medical need to further improve antimycotic therapy. We believe that based on our ever expanding knowledge base, interdisciplinary research bringing together chemical, molecular and clinical expertise can help make the antimycotic drugs of tomorrow fulfill the promise of today.

\section{ACKNOWLEDGEMENT}

K.T. acknowledges the receipt of a postdoctoral fellowship from the Industrial Research Fund (K. U. Leuven).

\section{REFERENCES}

[1] Nosanchuk JD. Current status and future of antifungal therapy for systemic mycoses. Recent Pat Anti-Infect Drug Discov 2006; 1:7584

[2] Fera MT, La Camera E, De Sarro A. New triazoles and echinocandins: mode of action, in vitro activity and mechanisms of resistance. Expert Rev Anti Infect Ther 2009; 7:981-98.

[3] De Sarro A, La Camera E, Fera MT. New and investigational triazole agents for the treatment of invasive fungal infections. J Chemother 2008; 20:661-71.

[4] Pasqualotto AC, Denning DW. New and emerging treatments for fungal infections. J Antimicrob Chemother 2008; 61:i19-30.

[5] Zhang AY, Camp WL, Elewski BE. Advances in topical and systemic antifungals. Dermatol Clin 2007; 25: 165-83.

[6] François IE, Aerts AM, Cammue BP, Thevissen K. Currently used antimycotics: spectrum, mode of action and resistance occurrence. Curr Drug Targets 2005; 6: 895-907.

[7] Rock FL, Mao W, Yaremchuk A, et al. An antifungal agent inhibits an aminoacyl-tRNA synthethase by trapping tRNA in the editing site. Science 2007; 316: 1759-61.

[8] Zirngibl L, Ed. Antifungal azoles: a comprehensive survey of their structures and properties. Weinheim: Wiley-VCH Verlag Gmbh, 1998.

[9] Maertens JA. History of the development of azole derivatives. Clin Microbiol Infec 2004; 10: 1-10.

[10] Heeres J, Backx LJ, Mostmans JH, Van Cutsem J. Griseofulvin: Antimycotic imidazoles. Part4. Synthesis and antifungal activity of ketoconazole, a new potent orally active broad spectrum antifungal agent. J Med Chem 1979; 22: 1003-5.

[11] Vanden Bossche H, Heeres J, Backx LJ, Marichal P, Willemsens G. Discovery, chemistry, mode of action and selectivity of itraconazole. In: Rippon JW, Fromtling RA, Eds. Cutaneous Antifungal Agents. New York: Marcel Dekker 1993: pp. 263-83.

[12] Richardson K. The discovery of fluconazole. In: Rippon JW, Fromtling RA, Eds. Cutaneous antifungal agents. New York: Marcel Dekker 1993: pp. 171-81. 
[13] Stütz A. The discovery and chemistry of Terbinafine. In: Rippon JW, Fromtling RA, Eds. Cutaneous antifungal agents. New York: Marcel Dekker 1993: pp. 117-26.

[14] Hartman PG, Polak A. Preclinical data of amorolfine. In: Rippon JW, Fromtling RA, Eds. Cutaneous antifungal agents. New York: Marcel Dekker 1993: pp. 13-26.

[15] Degreef $\mathrm{H}$, Heeres J, Borgers $\mathrm{M}$. Antifungal azoles for skin disorders. Exp Opin Ther Patents 2006; 16: 1235-53.

[16] Weinstein A, Berman B. Topical treatment of common superficial tinea infections. Am Fam Physician 2002; 65: 2095-102.

[17] Gupta AK, Ryder JE, Chow M, Cooper, EA. Dermatophytosis: the management of fungal infections. Skinmed 2005; 4: 305-10.

[18] Seebacher C, Brasch J, Abeck D, et al. German Society of Dermatology; German-speaking Mycological Society. Onychomycosis. Mycoses 2007; 50: 321-7.

[19] Lecha M, Effendy I, Feuilhade de Chauvin M, Di Chiacchio N, Baran R. Taskforce on Onychomycosis Education. Treatment options-development of consensus guidelines. J Eur Acad Dermatol Venerol 2005; 19: 25-33.

[20] Huang DB, Ostrosky-Zeichner L, Wu JJ, Pang KR, Tyring SK. Therapy of common superficial fungal infections. Dermatol Ther 2004; 17: 517-22.

[21] Borgers M, Degreef H, Cauwenbergh G. Fungal infections of the skin: infection process and antimycotic therapy. Curr Drug Targets 2005; 6: 849-62.

[22] Jesudanam TM, Rao GR, Lakshmi DJ, Kumari GR. Onychomycosis: a significant medical problem. Indian J Dermatol Venereol Leprol 2002; 68: 326-9.

[23] Hinojosa JR, Hitchcock K, Rodriguez JE. Clinical inquiries: which oral antifungal is best for toenail onychomycosis? J Fam Pract 2007; 56: 581-82.

[24] Baran R, Kaoukhov A. Topical antifungal drugs for the treatment of onychomycosis: an overview of current strategies for monotherapy and combination therapy. J Eur Acad Dermatol 2005; 19: 21-9.

[25] Frankum LE, Nightengale B, Russo M, Sarnes M. Pharmacoeconomic analysis of sequential treatment pathways in the treatment of onychomykosis. Manage Care Interface 2005; 18: 55-63.

[26] Avner S, Nir N, Henri T. Combination of oral terbinafine and topical ciclopirox compared to oral terbinafine for the treatment of onychomycosis. J Dermatol Treat 2005; 16: 327-30.

[27] Gupta AK, Schouten JR, Lynch LE. Ciclopirox nail lacquer 8\% for the treatment of onychomycosis: a Canadian perspective. Skin Ther Lett 2005; 10: 1-3.

[28] Baker SJ, Zhang YK, Akama T, et al. Discovery of a new boroncontaining antifungal agent, 5-fluoro-1,3-dihydro-1-hydroxy-2,1benzoxaborole (AN2690), for the potential treatment of onychomycosis. J Med Chem 2006; 49: 4447-50.

[29] Hui X, Baker SJ, Wester RC, et al. In vitro penetration of a novel oxaborole antifungal (AN2690) into the human nail plate. J Pharm Sci 2007; 96: 2622-31.

[30] Barak O, Loo, DS. AN-2690, a novel antifungal for the topical treatment of onychomycosis. Curr Opin Investig Drugs 2007; 8: 662-8.

[31] Pfaller MA, Boyken L, Hollis RJ, Messer SA, Tendolkar S, Diekema DJ. In vitro susceptibilities of Candida spp. to caspofungin: four years of global surveillance. J Clin Microbiol 2006; 44: 760-3.

[32] Marr KA. Invasive Candida infections: the changing epidemiology. Oncology (Williston Park). 2004; 18: 9-14.

[33] Marr KA, Carter RA, Crippa F, Wald A, Corey L. Epidemiology and outcome of mould infections in hematopoietic stem cell transplant recipients. Clin Infect Dis 2002; 34: 909-17.

[34] Lin X. Cryptococcus neoformans: morphogenesis, infection, and evolution. Infect Genet Evol 2009; 9: 401-16.

[35] Richardson M, Lass-Flörl C. Changing epidemiology of systemic fungal infections. Clin Microbiol Infect 2008; 14: 5-24.

[36] Morris A, Wei K, Afshar K, Huang L. Epidemiology and clinical significance of pneumocystis colonization. J Infect Dis 2008; 197: 10-17.

[37] Kauffman CA. Histoplasmosis: a clinical and laboratory update. Clin Microbiol Rev 2007; 20: 115-32.

[38] de Camargo ZP. Serology of paracoccidioidomycosis. Mycopathologia 2008; 165: 289-302.

[39] Laniado-Laborin R. Expanding understanding of epidemiology of coccidioidomycosis in the Western hemisphere. Ann N Y Acad Sci 2007; 1111: 19-34.
[40] Diekema DJ, Pfaller MA. Nosocomial candidemia: an ounce of prevention is better than a pound of cure. Infect Control Hosp Epidemiol 2004; 25: 624-6.

[41] Eggimann P, Garbino J, Pittet D. Epidemiology of Candida species infections in critically ill non-immunosuppressed patients. Lancet Infect Dis 2003; 3: 685-702.

[42] Minari A, Husni R, Avery RK, et al. The incidence of invasive aspergillosis among solid organ transplant recipients and implications for prophylaxis in lung transplants. Transpl Infect Dis 2002; 4: 195-200.

[43] Enoch DA, Ludlam HA, Brown NM. Invasive fungal infections: review of epidemiology and management options. J Med Microbiol 2006; 5: 809-18.

[44] Ostrosky-Zeichner L, Rex JH, Pappas PG, et al. Antifungal susceptibility survey of 2,000 bloodstream Candida isolates in the United States. Antimicrob Agents Chemother 2003; 47: 3149-54.

[45] Bicanic T, Wood R, Meintjes G, et al. High-dose amphotericin B with flucytosine for the treatment of cryptococcal meningitis in HIV-infected patients: a randomized trial. Clin Infect Dis 2008; 47: 123-30.

[46] Ellis D. Amphotericin B: spectrum and resistance. J Antimicrob Chemother 2002; 49: 7-10.

[47] Petrikkos GL. Lipid formulations of amphotericin B as first-line treatment of zygomycosis. Clin Microbiol Infect 2009; 15: 87-92.

[48] Slørdal, L, Spigset O. Heart failure induced by non-cardiac drugs. Drug Saf 2006; 29; 567-86.

[49] Diekema DJ, Messer SA, Hollis RJ, Jones RN, Pfaller MA. Activities of caspofungin, itraconazole, posaconazole, ravuconazole, voriconazole, and amphotericin B against 448 recent clinical isolates of filamentous fungi. J Clin Microbiol 2003; 41: 3623-6.

[50] Hoffman HL, Rathbun RC. Review of the safety and efficacy of voriconazole. Expert Opin Investig Drugs 2002; 11:409-29.

[51] Maertens J, Meersseman W, Van Bleyenbergh P. New therapies for fungal pneumonia. Curr Opin Infect Dis 2009; 22:183-90.

[52] Sabatelli F, Patel R, Mann PA, et al. In vitro activities of Posaconazole, Fluconazole, Itraconazole, Voriconazole, and Amphotericin B against a large collection of clinically important molds and yeasts. Antimicrob Agents Chemother 2006; 50: 200915 .

[53] Denning DW. Echinocandin antifungal drugs. Lancet 2003; 362 : 11423-51.

[54] Pappas PG, Rex JH, Sobel JD, et al. Infectious Diseases Society of America. On behalf of the Infectious Diseases Society of America: Guidelines for the treatment of candidiasis. Clin Infect Dis 2004; 38: 161-89.

[55] Ullmann AJ, Cornely OA. Antifungal prophylaxis for invasive mycoses in high risk patients. Curr Opin Infect Dis 2006; 19: 57176

[56] Manavathu EK, Cutright JL, Chandrasekar PH. Organismdependent fungicidal activities of azoles. Antimicrob Agents Chemother 1998; 42: 3018-21

[57] Morris MI, Villmann M. Echinocandins in the management of invasive fungal infections, Part 2. Am J Health Syst Pharm 2006; 63: 1813-20.

[58] Manavathu EK, Ramesh MS, Baskaran I, Ganesan LT, Chandrasekar PH. A comparative study of the post-antifungal effect (PAFE) of amphotericin B, triazoles and echinocandins on Aspergillus fumigatus and Candida albicans. J Antimicrob Chemother 2004; 53: 386-9.

[59] Vanden Bossche H. Biochemical targets for antifungal azole derivatives: hypothesis on the mode of action. In McGinnis MR, Ed. Current topics in medical mycology. New York: Springer Verlag 1985; 1: pp. 313-59.

[60] De Nollin S, Van Belle H, Goossens F, Thone F, Borgers M. Cytochemical and biochemical studies of yeast after in vitro exposure to miconazole. Antimicrob Agents Chemother 1977; 11: 500-13.

[61] Kobayashi D, Kondo K, Uehara N, et al. Endogenous reactive oxygen species is an important mediator of miconazole antifungal effect. Antimicrob Agents Chemother 2002; 45: 3113-7.

[62] François IE, Cammue B, Borgers M, Ausma J, Dispersyn GD, Thevissen K. Azoles: Mode of antifungal action and resistance development: effect of miconazole on endogenous reactive oxygen species production in Candida albicans. Anti-Infective Agents in Med Chem 2006; 5: 3-13. 
[63] Thevissen K, Ayscough KR, Aerts AM, et al. Miconazole induces changes in actin cytoskeleton prior to reactive oxygen species induction in yeast. J Biol Chem 2007; 282: 21592-7.

[64] Hornby JM, Nickerson, KW. Enhanced production of farnesol by Candida albicans treated with four azoles. Antimicrob Agents Chemother 2004; 48: 2305-7.

[65] Phillips AJ, Sudbery I, Ramsdale M. Apoptosis induced by environmental stresses and amphothericin B in Candida albicans. Proc Natl Acad Sci USA 2003; 100: 14327-32.

[66] Sud IJ, Feingold DS. Action of antifungal imidazoles on Staphylococcus aureus. Antimicrob Agents Chemother 1982; 22: 470-4.

[67] Faergemann J. Management of seborrhoeic dermatitis and pityriasis versicolor. Am J Clin Dermatol 2000; 1: 75-80.

[68] Edlind T, Smith L, Henry K, Katiyar S, Nickels J. Antifungal activity in Saccharomyces cerevisiae is modulated by calcium signalling. Mol Microbiol 2002; 46: 257-68.

[69] Onyewu C, Blankenship JR, Del Poeta M, Heitman J. Ergosterol biosynthesis inhibitors become fungicidal when combined with calcineurin inhibitors against Candida albicans, Candida glabrata, and Candida krusei. Antimicrob Agents Chemother 2003; 47: 95664.

[70] Vanden Bossche H. Inhibitors of P450-dependend steroid biosynthesis: from research to medical treatment. J Steroid Biochem 1992; 43: 1003-21.

[71] Vanden Bossche H, Bellens W, Cools J, et al. Cytochrome P450: target for itraconazole. Drug Dev Res 1986; 8: 287-98.

[72] Lamb DC, Kelly DE, Baldwin BC, Kelly SL. Differential inhibition of human CYP3A4 and Candida albicans CYP51 with azole antifungal agents. Chem Biol Interact 2000; 125: 165-75.

[73] Sakaeda T, Iwaki K, Kakumoto M, et al. Effect of micafungin on cytochrome $\mathrm{P} 450 \quad 3 \mathrm{~A} 4$ and multidrug resistance protein 1 activitities, and its comparison with azole antifungal drugs. J Pharm Pharmacol 2005; 57: 759-64.

[74] Venkatakrishnan K, Von Moltke LL, Greenblatt DJ. Effects of the antifungal agents on oxidative drug metabolism: clinical relevance. Clin Pharmacokinet 2000; 38: 111-80.

[75] Lee CR, Goldstein GA, Pieper JA. Cytochrome P450 2C9 polymorphisms: a comprehensive review of the in-vitro and human data. Pharmacogenetics 2002; 12: 251-63.

[76] Parker JE, Merkamm M, Manning NJ, Pompon D, Kelly SL, Kelly DE. Differential azole antifungal efficacies contrasted using a Saccharomyces cerevisiae strain humanized for sterol 14 alphademethylase at the homologous locus. Antimicrob Agents Chemother 2008; 52:3597-603.

[77] Meletiadis J, Chanock S, Walsh TJ. Defining targets for investigating the pharmacogenomics of adverse drug reactions to antifungal agents. Pharmacogenomics 2008; 9: 561-84.

[78] Sanglard D, Bille J. Current understanding of the modes of action of and resistance mechanisms to conventional and emerging antifungal agents for treatment of Candida infections. In: Calderone R, Ed. Candida and candidiasis. USA: ASM Press, 2002: pp. 34983.

[79] Pinjon E, Moran GP, Jackson CJ, et al. Molecular mechanisms of itraconazole resistance in Candida dubliniensis. Antimicrob Agents Chemother 2003; 47: 2424-37.

[80] Henry KW, Nickels JT, Edlind TD. Upregulation of ERG genes in Candida species by azoles and other sterol biosynthesis inhibitors. Antimicrob Agents Chemother 2000; 44: 2693-700.

[81] Chau AS, Mendrick CA, Sabatelli FJ, Loebenberg D, McNicholas PM. Application of real-time quantitative PCR to molecular analysis of Candida albicans strains exhibiting reduced susceptibility to azoles. Antimicrob Agents Chemother 2004; 48: 2124-31.

[82] Sanguinetti M, Posteraro B, Fiori B, Ranno S, Torelli R, Fadda G. Mechanisms of azole resistance in clinical isolates of Candida glabrata collected during a hospital survey of antifungal resistance. Antimicrob Agents Chemother 2004; 49: 668-79.

[83] Vermitsky JP, Edlind TD. Azole resistance in Candida glabrata: coordinate upregulation of multidrug transporters and evidence for a Prd1-like transcription factor. Antimicrob Agents Chemother 2004; 48: 3773-81.

[84] Kelly SL, Lamb DC, Kelly DE. Y132H substitution in Candida albicans sterol 14alpha-demethylase confers fluconazole resistance by preventing binding to haem. FEMS Microbiol Lett 1999; 180: 171-5.
[85] Kelly SL, Lamb DC, Loeffer J, Einsele H, Kelly DE. The G464S amino acid substitution in Candida albicans sterol 14alphademethylase causes fluconazole resistance in the clinic through reduced affinity. Biochem Biophys Res Commun 1999; 262: 174-9.

[86] Costeron JW, Cheng KJ, Geesey GG, et al. Bacterial biofilms in nature and disease. Annu Rev Microbiol 1987; 41: 435-64.

[87] Donlan RM. Biofilms: microbial life on surfaces. Emerg Infect Dis 2002; 8: 881-90.

[88] Khardori N, Yassien M. Biofilms in device-related infections. J Ind Microbiol 1995; 15: 141-7.

[89] Costerton JW, Stewart PS, Greenberg EP. Bacterial biofilms: a common cause of persistent infections. Science 1999; 284: 1318 22.

[90] Donlan RM. Biofilms and device-associated infections. Emerg Infect Dis 2001; 7: 277-81

[91] Donlan RM. Biofilm formation: a clinically relevant microbiological process. Clin Infect Dis 2001; 33: 1387-92.

[92] Donlan RM, Costerton RW. Biofilms: survival mechanisms of clinically relevant microorganisms. Clin Microbiol Rev 2002; 15: 167-93.

[93] Kojic EM, Darouiche, RO. Candida infections of medical devices. Clin Infect Dis 2004; 17: 255-67.

[94] Douglas LJ. Medical importance of biofilms in Candida infections. Rev Iberoam Micol 2002; 19: 139-43.

[95] Ramage G, Saville SP, Thomas DP, López-Ribot JL. Candida biofilms: an update. Eukaryot Cell 2005; 4: 633-8.

[96] Douglas JL. Candida biofilms and their role in infection. Trends Microbiol 2003; 11: 30-6.

[97] Kuhn DM, Ghannoum MA. Candida biofilms: antifungal resistance and emerging therapeutics options. Curr Opin Investig Drugs 2004; 5: 186-97.

[98] Baillie GS, Douglas LJ. Matrix polymers of Candida biofilms and their possible role in biofilm resistance to antifungal agents. J Antimicrob Chemother 2000; 46: 397-403.

[99] Mukherjee PK, Chandra J, Kuhn DM, Ghannoum MA. Mechanism of fluconazole resistance in Candida albicans biofilms: phasespecific role of efflux pumps and membrane sterols. Infect Immun 2003; 71: 4333-40.

[100] Ramage G, VandeWalle K, Bachmann SP, Wickes BL, LópezRibot JL. In vitro pharmacodynamic properties of three antifungal agents against preformed Candida albicans biofilms determined by time-kill studies. Antimicrob Agents Chemother 2002; 46: 3634-36.

[101] Ramage G, Bachmann S, Patterson TF, Wickes BL, López-Ribot JL. Investigation of multidrug efflux pumps in relation to fluconazole resistance in Candida albicans biofilms. J Antimicrob Chemother 2002; 49: 973-80.

[102] Al-Fattani MA, Douglas LJ. Penetration of Candida albicans biofilms by antifungal agents. Antimicrob Agents Chemother 2004; 48: 3291-7.

[103] Mateus C, Crow SA, Jr, Ahearn DG. Adherence of Candida albicans to silicone induces immediate enhanced tolerance to fluconazole. Antimicrob Agents Chemother 2004; 48: 3358-66.

[104] Kumamoto CA. A contact-activated kinase signals Candida albicans invasive growth and biofilm development. Proc Natl Acad Sci USA 2005; 102: 5576-81.

[105] Verweij PE, Howard SJ, Melchers WJ, Denning DW. Azoleresistance in Aspergillus: proposed nomenclature and breakpoints. Drug Resist Updat 2009; 12: 141-7.

[106] Perlin DS. Resistance to echinocandin-class antifungal drugs. Drug Resist Updat 2007; 10: 121-30.

[107] Bellmann R. Clinical pharmacokinetics of systemically administered antimycotics. Curr Clin Pharmacol 2007; 2: 37-58.

[108] Aperis G, Mylonakis E. Newer triazole antifungal agents: pharmacology, spectrum, clinical efficacy and limitations. Expert Opin Investig Drugs 2006; 15: 579-602.

[109] Odds F, Ausma J, Van Gerven F, et al. In vitro and in vivo activity of the novel azole antifungal agent R126638. Antimicrob Agents Chemother 2004; 48: 388-91.

[110] Rex JH, Pfaller MA. Has antifungal suscebtibility testing come of age? Clin Infect Dis 2002; 35: 982-9.

[111] Arikan S. Current status of antifungal susceptibility testing methods. Med Mycol 2007; 45: 569-87.

[112] Cantón E, Espinel-Ingroff A, Pemán J. Trends in antifungal susceptibility testing using CLSI reference and commercial methods. Expert Rev Anti Infect Ther 2009; 7: 107-19. 
[113] Serefko A, Los R, Biernasiuk A, Malm A. Comparison of microdilution method and E-test procedure in susceptibility testing of caspofungin against Candida non-albicans species. New Microbiol 2008; 31: 257-62.

[114] De Brabander M, Aerts F, Van Cutsem J, van den Bossche H, Borgers $M$. The activity of ketoconazole in mixed cultures of leukocytes and Candida albicans. Sabouraudia 1980; 18: 197-210.

[115] Cetinkaya Z, Kiraz N, Karaca S, et al. Antifungal susceptibilities of dermatophytic agents isolated from clinical specimens. Eur J Dermatol 2005; 5: 258-61.

[116] Osborne CS, Leitner I, Favre B, Ryder NS. Antifungal drug response in an in vitro model of dermatophyte nail infection. Med Mycol 2004; 42: 159-63.

[117] Borgers M. Ultrastructural correlates of antimycotic treatment. In: McGinnis MR Ed. Current topics in medical mycology. New York: Springer-Verlag 1988: pp. 1-39.

[118] Morrison VA. Caspofungin: on overview. Expert Rev Anti Infect Ther 2005; 3: 697-705.

[119] Ikeda F, Tanaka S, Ohki H, et al. Role of micafungin in the antifungal armamentarium. Curr Med Chem 2007; 14: 1263-75.

[120] Vehreschild JJ, Cornely OA. Micafungin sodium, the second of the echinocandin class of antifungals: theory and practice. Future Microbiol 2006; 1: 161-70.

[121] Vazquez JA, Sobel JD. Anidulafungin: a novel echinocandin. Clin Infect Dis 2006; 43: 215-22.

[122] Kofla G, Ruhnke M. Voriconazole: review of a broad spectrum triazole antifungal agent. Expert Opin Pharmacother 2005; 6: 121529.

[123] Hof H. A new broad-spectrum azole antifungal: posaconazolemechanisms of action and resistance, spectrum of activity. Mycoses 2006; 49: 2-6.

[124] Chen A, Sobel JD. Emerging azole antifungals. Expert Opin Emerg Drugs 2005; 10: 21-33.

[125] Baran R, Gupta AK, Pierard GE. Pharmacotherapy of onychomycosis. Expert Opin Pharmacother 2005; 6: 609-24.

[126] Gupta AK, Ryder JE, Summerbell RC. Onychomycosis: classification and diagnosis. J Drugs Dermatol 2004; 3: 51-6.

[127] Gupta AK, Tomas E. New antifungal agents. Dermatol Clin 2003; 21: $565-76$.

[128] Fung-Tomc JC, Huczko E, Minassian B, Bonner DP. In vitro activity of a new oral triazole, BMS-207147 (ER-30346). Antimicrob Agents Chemother 1998; 42: 313-8.
[129] Piérard-Franchimont C, Ausma J, Wouters L, et al. Activity of the triazole antifungal r126638 as assessed by corneafungimetry. Skin Pharmacol Physiol 2006; 19: 50-6.

[130] Piérard GE, Ausma J, Henry F, et al. A pilot study on seborrheic dermatitis using pramiconazole as a potent oral anti-Malassezia agent. Dermatology 2007; 214: 162-9.

[131] Donders G, Ausma J, Wouters L, Cauwenbergh G, Borgers M, Janssens D. Efficacy of a single oral dose of $200 \mathrm{mg}$ pramiconazole in vulvovaginal yeast infections: an exploratory phase IIa trial. Acta Derm Venereol 2008; 88: 462-6.

[132] Decroix J, Ausma J, Cauwenbergh G, Borgers M, Wouters L. The efficacy of oral treatment with pramiconazole in tinea pedis and tinea cruris/corporis: two exploratory phase IIa trials. $\mathrm{Br} J$ Dermatol. 2008; 158: 854-6.

[133] Pasqualotto Pasqualotto AC, Denning DW. New and emerging treatments for fungal infections. J Antimicrob Chemother 2008; 61: i19-30.

[134] Morris MI. Posaconazole: a new oral antifungal agent with an expanded spectrum of activity. Am J Health Syst Pharm 2009; 66: 225-36.

[135] Kamai Y, Harasaki $\mathrm{T}$, Fukuoka $\mathrm{T}$, et al. In vitro and in vivo activities of CS-758 (R-120758), a new trizole antifungal agent. Antimicrob Agents Chemother 2002; 46: 367-70.

[136] Kamai Y. CS-758, a new triazole agent: In vivo antifungal activity against experimental candidiasis, aspergillosis, and cryptococcosis Ann Rep Sankyo Res Lab 2004; 56: 1-10.

[137] Borelli C, Schaller M, Niewerth M, et al. Modes of action of the new arylguanidine abafungin beyond interference with ergostero biosynthesis and in vitro activity against medically important fungi. Chemotherapy 2008; 54: 245-59.

[138] Uchida K, Nishiyama Y, Tanaka T, Yamaguchi H. In vitro activity of novel imidazole antifungal agent NND-502 against Malassezia species. Int J Antimicrob Agents 2003; 21: 234-38.

[139] Uchida K, Nishiyama $\mathrm{Y}$, Yamaguchi $\mathrm{H}$. In vitro activity of luliconazole (NND-502), a novel imidazole antifungal agent. J Infect Chemother 2004; 10: 216-19.

[140] Wess L, Haan K. Cracking antifungals. Biocentury 2004; 12: 1-20.

[141] Karwa R, Wargo KA. Efungumab: a novel agent in the treatment of invasive candidiasis. Ann Pharmacother 2009; 43: 1818-23. 